\title{
"Basic Sciences for Medicine": A basic-research program of the Russian Academy of Sciences Presidium
}

\author{
Grigoryev A.I. \\ Program Coordinator \\ Doctor of Medical Sciences, Member of the Russian Academy of Sciences (RAS) and Russian \\ Academy of Medical Sciences (RAMS), Vice-President of RAS.
}

$\mathrm{T}$ he RAS Presidium program for basic research "Basic Sciences for Medicine" was started in 2002 and is approved annually by the RAS Presidium. Over the 8 years of its existence, this program has demonstrated that it is an essential and effective part of RAS innovation activities. It is an interdisciplinary program conducted annually on a competitive basis. The aim of the program is to use the results of basic research obtained in exact and natural sciences in order to solve the high-priority practical problems of healthcare, especially those under research in RAS medical facilities. The main aims are (a) to study the etiology and pathogenesis of a variety of widespread and socially important diseases; (b) to develop new medical technologies such as diagnostics, medical equipment, and drugs; (c) to encourage RAS medical facilities in their attempts to develop new technologies and conduct clinical trials for these technologies; (d) to introduce tested results into the practice of RAS medical facilities and into the Russian Healthcare system as a whole; and (e) to increase the quality of medical services provided to RAS employees. The WHO predicts that over the next 1015 years, neurological and psychological conditions will be the most common form of illness, overtaking the cardiovascular diseases and cancer which are currently dominant. Taking this into account, a new section has been created inside this program in 2010 to stimulate brain research.

The importance of this program is based on its wide possibilities for social use and its unique ideology aimed at introducing the achievements of exact and natural sciences such as biology, chemistry, physics, and mathematics into practical medicine by pooling a major part of the resources of RAS-affiliated scientific research institutes.

The multidisciplinary proficiency of RAS institutes and their high scientific and staff potential allow a very wide scope of research and implementation activities, starting with obtaining deeper or new knowledge on the etiology and pathogenesis of socially-important diseases and ending with the development and manufacture of new drugs or materials. Moreover, the unique technical and material base of RAS facilities specializing in physics makes the construction of medical equipment and its adaptation for mass production possible. One of the tactical objectives of this program is to fund projects which can be practically carried out in the course of a few years.

\section{THE SCIENTIFIC ORGANIZATIONAL STRUCTURE OF THE PROGRAM}

The scientific organizational management of the program is provided by the coordinator and the scientific council, which is headed by a chairman appointed by the coordinator (Fig. 1). The scientific council includes leading scientists, mainly members of RAS and RAMS.

The coordinator and the scientific council for the ideology of the program's development, selects projects to be funded, establishes the extent of the funding, and assesses the results obtained during the course of the projects. The work of the coordinator and the scientific council is mostly based on the opinions of the expert council, which they appoint. This council includes leading scientists from RAS, RAMS, the Ministry of Healthcare and Social Development, and other organizations. This council performs an expert assessment of projects submitted for the competition and the results obtained by the funded projects during the course of the year, firstly as sectional moderators on the annual program conferences and secondly as reviewers of the annual reports. The connection between the coordinator and, on one hand, the scientific council and, on the other, scientific organizations is the coordination center (Fig. 1). Its functions are the following:

(i) conducting the competition,

(ii) organizing the annual reporting conference,

(iii) collecting annual project reports, (iv) reporting the Program's activity in the media and on the program's website. 


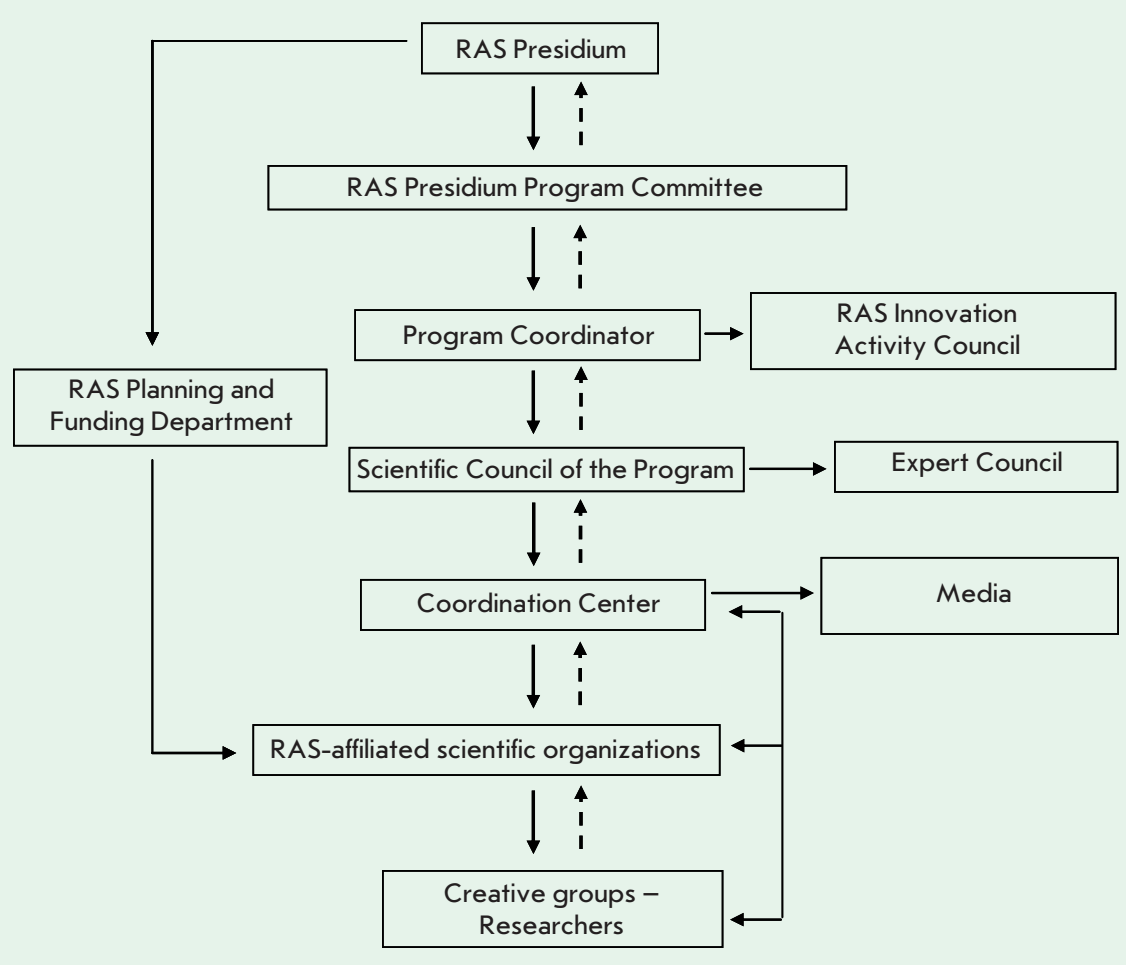

Fig. 1. Schematic of the organization of the "Basic Science for Medicine" Program

The program is developing very quickly and exhibiting very dynamic growth. For example, in 2002-2003, research was conducted in only three fields:

(i) A study of the etiology and pathogenesis of widespread and socially important diseases;

(ii) The development and improvement of diagnostic methods and equipment;

(iii) The development of medical materials and equipment and new drugs.

In 2004, a new line of research was introduced under the title Current Problems of Radiobiology; within the original fields, the most promising projects concerning chronic neurodegenerative diseases (Parkinson's disease, Alzheimer's disease, etc.), biophotonics, and informatics were formed. As of 2005, a number of innovation projects have been joined into groups which are now the basis for trials and implementation of new medical technologies in RAS medical facilities; the RAS Central Clinical Hospital; and other RAS hospitals for scientific centers in St. Petersburg, Pushino, Chernogolovka, Troitsk, Ka- zan, and the Kola Scientific Research Center.

A new line was also formed in 2006 under the title Human Polymorphisms. It was developed and financed in conjunction with Moscow State University. Finally, in 2010, in accordance with a decree from the RAS General Assembly (dated December 2009), a group of projects concerning brain research was started. The further development of the program has the virtually unlimited resources of RAS institutes at its disposal, but unfortunately many tens of projects have to be declined due to the limited funding of the Program, even though these projects are of high theoretical and practical interest.

The dynamic development of the program is easily quantified. From 2002 to 2010 , the number of funded projects has increased from 37 to 187 , which is 5-fold; the number of organizations taking part in the projects has increased from 20 to 72 (3.5-fold); and the funding has increased from 30 to 100 million rubles (over 3 -fold) (Fig. 2). Currently eight branches of the RAS are taking part in the program in many Russian cities (Moscow, St. Petersburg, Pushino, Nizhniy Novgorod, Samara, Kazan, Chernogolvka, and Ufa). Taking into account the innovational direction of the program, one of the key indexes of its effectiveness is the number of patent applications and granted patents. In 2002-2010 these indexes have increased more than tenfold (Fig. 3).

One of the most important elements in the program of the development of the Central Region branch of the RAS was the creation of branch offices in the regional centers of the RAS (Siberian, Ural, and Far East centers).

As was mentioned earlier, the results of the program's work are summarized annually during conferences which take place first in all the branch offices of RAS starting with the Far East, Siberian, and the Ural RAS regional branches; then they are followed by a joint conference that includes all the institutes of the Central Region branch of the RAS. A new approach was taken in 2009: the conferences were organized according to specialization. These conferences attract a lot of interest from the scientific and medical communities, and the conferences are always summarized at special press conferences for media such as ITAR-TASS, Meditsinskaya Gazeta (Medical Gazette), Poisk (Search), Radio Rossii (Radio Russia), TV media, etc.

\section{A SHORT SUMMARY OF THE PROGRAM'S RESULTS}

\section{BASIC RESEARCH INTO THE ETIOLOGY AND PATHOGENESIS OF WIDESPREAD AND SOCIALLY IMPORTANT DISEASES}

The biggest progress in this line of research has been achieved in the study of the pathogenesis and etiology of neurodegenerative diseases (NDD).

NDDs include Parkinson's disease, Alzhemier's disease, hyperlactatemia, etc., all of which result in the death of certain neuron populations. These diseases are currently as common as cardiovascular and oncological diseases. Neuronal death causes a deficiency in the amount of chemical signals synthesized by these cells and disrupts the functions which they regulate. The first symptoms of an NDD usually emerge after the death of most of a certain population of neurons. This process usually 
$\square$ Number of projects $\square$ Number of organizations Funding (millions of rubles)

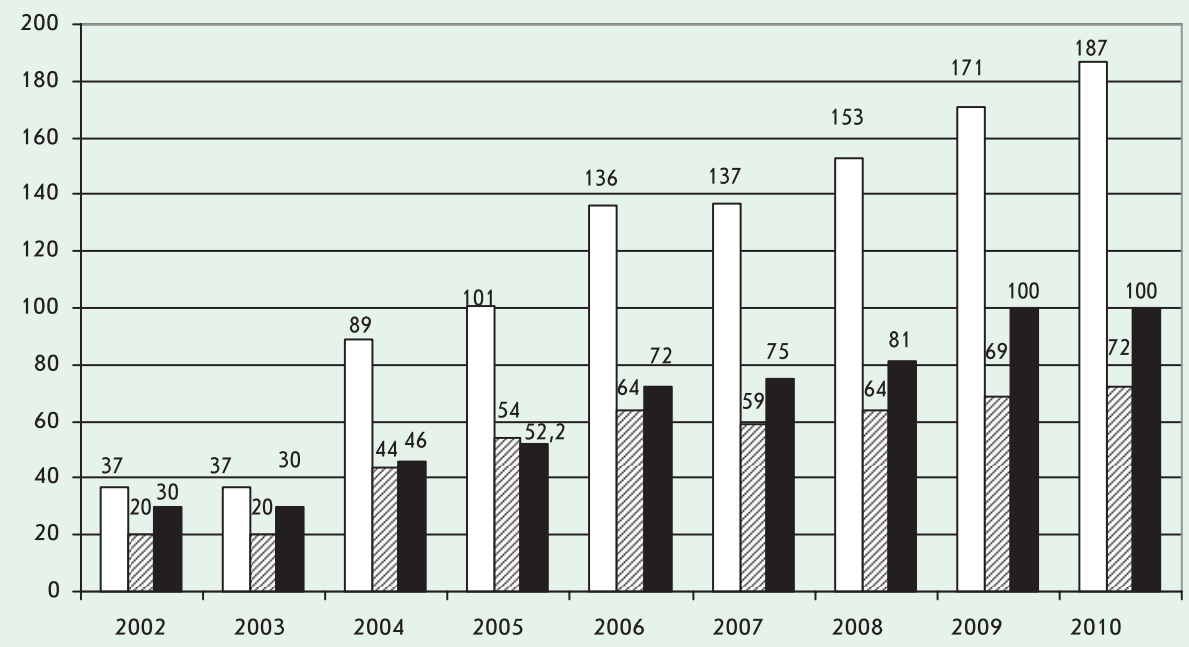

Fig. 2. Major indicators of the Program's development

begins at the age of $25-35$ and can continue for 20-30 years. Treatment usually begins only after the emergence of symptoms and does not lead to patient being cured, so the patient gradually becomes incapacitated and dies.

A project supported by the program showed for the first time that a deficit of signal molecules caused by the death of the neurons that synthesized these molecules was compensated by changes in the synthesizing pathways of other neurons, which allowed the additional synthesis of these signals and explained such a long asymptomatic period for NDDs (Koltsov Institute of Developmental Biology, RAS).

In this context, the emergence of symptoms indicates the irreversible destruction of the relevant regulatory system and the depletion of the compensating mechanisms. These factors explain the ineffectiveness of current treatments. This creates a rationale for developing NDD diagnostics, which could identify the disease long before the emergence of symptoms, as well as for prophylactic treatment, which would stop or at least delay the death of neurons.

New and important data were also obtained in the course of studying the etiology and pathogenesis of other socially important diseases, such as lung and cardiovascular deficiency, diabetes, and tuberculosis.

\section{DEVELOPMENT AND IMPROVEMENT OF DIAGNOSTICS AND TREATMENT}

Diagnostics. New and improved diagnostic and monitoring methods for a wide range of neurological, endocrine, cardiovascular, lung, and digestive conditions have also been developed. These studies were successful due to the focus on the metabolic dysfunctions in the damaged organs and tissues. Such dysfunctions can be detected by testing for specific endogenous
Fig. 3. Major indicators of the Program's effectiveness markers. One of the most important priorities of this line of research is the development of noninvasive diagnostic approaches. Among these, biophotonics seem to be very promising, including termography, endofluorescence, and coherent optical tomography, all of which seem to be especially promising for the diagnosis of oncological conditions.

Thus, researchers working with support from the program created and implemented a noninvasive diagnostic method based on coherent tomography, which makes targeted biopsy, identifying the borders of the pathological zone during operations, and monitoring wound healing possible. A portable, high-resolution tomographic scanner and microscope were created for use with this method at the Institute of Applied Physics, RAS.

Studies of NDD pathologies conducted within the program allowed researchers to understand the importance of preclinical diagnostic methods (see earlier). It is clear that a presymtomatic diagnosis can be made based on positron-emission tomography. This method is expensive and is therefore hardly accessible during a large-scale prophylactic medical examination; however it can be used for diagnosing patients from a small risk group. For this, researchers involved in the Program are searching for peripheral markers of NDD both in

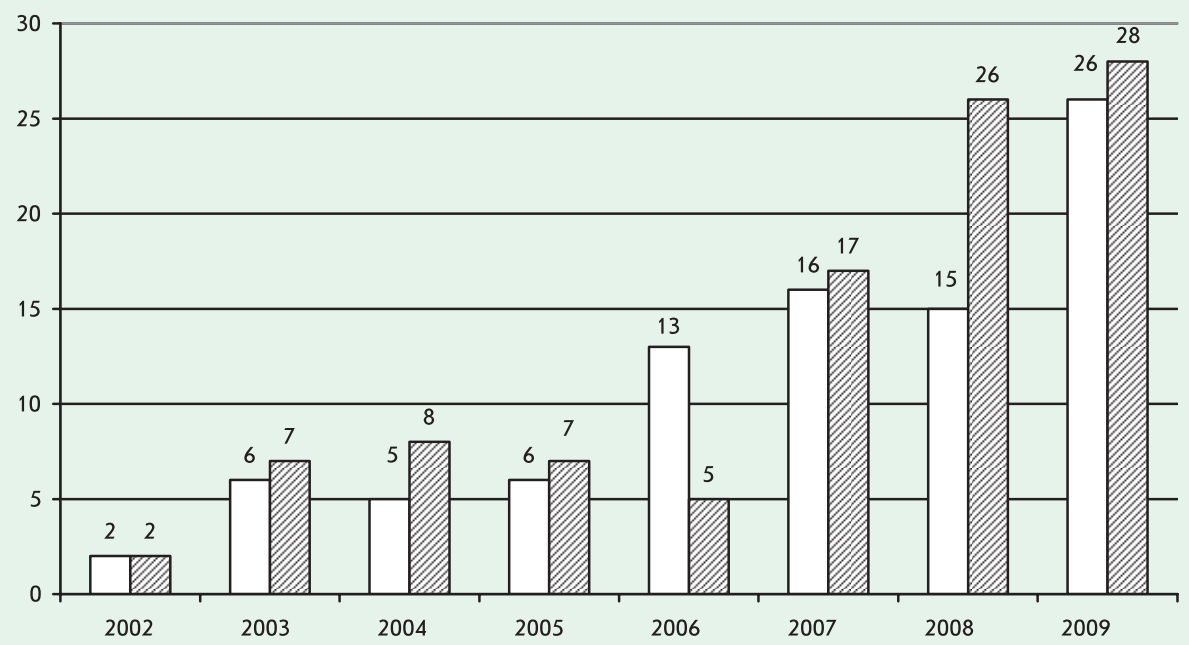


experimental systems and in patients who have only recently started manifesting symptoms.

In the future this work may make it possible to identify risk groups based on the results of a routine large-scale prophylactic examination (Koltsov Institute of Developmental Biology, RAS, and the Central Clinical Hospital of the RAS). The development of preclinical diagnostics for NDD will open new possibilities for the development of prophylactic therapy.

One of the achievements of the program is the development of an early-stage diagnostic method for eye diseases based on the appearance of arestin, a protein involved in the phototransduction cycle. It has also been demonstrated that the detection of antibodies against the S-antigen, one of the retina proteins, in the blood correlates with the development of diabetic retinopathy in diabetes patients and posttraumatic complications in eye burn trauma (Biochemical Physics Institute, RAS).

In recent years, new informatics approaches that can increase the predictive power of diagnostics for a wide range of diseases have been developed. The creation of a basic telemedical network for patients and prophylactic centers (Fig. 6), including mobile functional diagnostics that can be performed at a distance (Institute of Biological Instrumentation, RAS), is of great importance. This network will be used for express diagnostics and for monitoring the patient's condition; it will involve the best specialists from Moscow and St. Petersburg.

The projects on the development of information technologies for supporting diagnoses and treatments and the creation of an integrated information system for medical facilities (based in the Central Clinical Hospital, RAS) have also been competed (The Research Center of Medical Informatics at the Institute of Program Systems, RAS).

Treatment. Based on the results of experiments that showed the selective effect of transcranial electrostimulation on the brain's protective systems (which react by releasing endorphin and serotonin), specialists developed a therapeutic method involving transcranial electrostimulation (TES therapy).
This method uses the surface electrodes and currents of a specific form to induce the activity of the brain's protective systems. This method can be used for treating a wide range of neurological conditions (Pavlov Institute of Physiology, RAS).

\section{THE CREATION OF MEDICAL EQUIPMENT, MATERIALS AND NEW DRUGS}

Equipment. In the course of our program, researchers have improved several methods of therapy by developing the necessary instrumentation, including that utilizing the effect of lasers on biological tissues. Some of the projects have moved into the clinical trial phase and some new drugs are being implemented. For instance, a periodicalimpulse CO2 laser was created for the treatment of skin diseases, such as for the dissection of papillomas and malformations and for the removal of burn marks (General Physics Institute, RAS). This device has completed clinical trials in the three leading dermatological clinics in Russia. It conforms to the best technical standards seen in similar devices around the world, but it costs about ten times less.

A compact infrared computer tomographic scanner with enhanced metrological capabilities has also been constructed (Institute of Radiotechnics and Electrotechnics, RAS). Using this device, a number of medical institutions have created infrared diagnostic facilities and devised new methods for the differential diagnosis of tumors, diseases of the vascular system, motor apparatus, and mammography. In particular, an infrared thermographic imager was manufactured with the help of the program and became the base for an infrared diagnostic facility in Clinic no. 2 at the Central Clinical Hospital of the RAS.

The widespread use of cryotherapy and cryosurgery in Russian practical medicine (dermatology, gynecology, otolaryngology, general surgery, oncology, and microsurgery) is obstructed by the absence of simple and reliable cryomedical instruments intended for rapid and contact-free freezing (liquid nitrogen sprays) and the deep contact freezing of select surfaces or volumes of tissue (active-tip cryodestructors with liquid nitrogen cooling).
The following has been achieved in the course of the Program: (a) a critical analysis of the practical experience in developing and using liquid nitrogen sprays and cryodestructors with interchangeable tips; (b) the modernization of portable cryoinstrument construction; (c) prototypes of portable cryosprays and cryodestructors have been manufactured (Institute of Solid State Physics of RAS) and are being tested in clinics at the scientific facilities of the RAS in Chernogolovka, Saint-Petersburg, and several clinical facilities in Moscow.

Materials. Researchers have obtained new results which pertain to the use of chemical or biological materials for replacing damaged tissues in the organism and for the endoprosthetics of joints and blood vessels. New matrix materials based on natural polysaccharides have been created for the cultivation of human skin cells, which can then be transplanted to facilitate the healing of wounds (the Institute of High Molecular Weight Compounds, RAS, and the RAS Cytology Institute). It has been demonstrated that the most promising polymer materials for the cultivation of human fibroblasts and keratinocytes are filmlike composite matrices formed by chitozan and modified by collagen. A method for perforating these filmlike matrices by a laser beam has also been developed.

Drugs. Research supported by the Program has yielded clinical experimental data which elicits the use of the Russian drug Xymedon for the prophylactic treatment of secondary immunodeficiency (Arbuzov Institute of Organic and Physical Chemistry, Kazan Scientific Center, RAS). This drug can normalize the somatic status of HIV patients by lowering HIV replication (viral load) and it can help improve the pathogenically relevant parameters of the immune system. Based on this, the criteria and schemes of drug administration have been fine tuned for the treatment of HIV infection. This is the first instance of treating HIV with a low-toxicity nonglycoside analog of pyrimidine nucleosides.

A novel group of original compounds for the treatment of Alzheimer's disease and other NDDs has also been created (Institute of Physiologically Active Compounds, RAS). 


\section{CURRENT PROBLEMS IN RADIOBIOLOGY}

Studies on the effects of high energy nuclei and low-intensity irradiation of living organisms have been conducted in several RAS institutes (Emanuel Institute of Biochemical Physics, Institute of Theoretical and Experimental Biophysics and the Semenov Institute of Chemical Physics). These studies have developed new technologies for the irradiation of living organisms, including some methods that lower the negative effect of irradiation therapy (Trapeznikov Instiute of Control Sciences, RAS). Studies have led to the creation of drugs of natural origins that can effectively decrease radiation-induced damage in human cells (Institute of Petrochemical Synthesis, RAS, and Konstantinov Institute of Nuclear Physics, St. Petersburg, RAS). Researchers from the Emanuel Institute of Biochemical Physics, RAS, have developed criteria for determining the resistance of a human organism to extreme conditions. Considerable efforts are being put in to construct new instrumentation, such as a new generation of dosimeters and an irradiation source (Prokhorov Institute of General Physics, RAS). This line has recently been reorganized and the projects have been included into other lines of research according to their subject.

\section{HUMAN POLYMORPHISM}

Projects in this line of research are involved in basic scientific research concerning the individual variety of biomacromolecules in humans at the genetic and protein levels. A comparative study has been performed in the field of structural functional and evolutionary genomics, including cloning, sequencing and an analysis of the polymorphism of the prepromotor region of the ribosomal DNA of humans and higher primates (Gene Biology Institute, RAS). Both similar and highly divergent regions have been found, the latter being due to single substitutions, insertions, deletions, and structural reorganizations. Several hypotheses on the initiating mechanisms of genome evolution were formulated. Researchers have suggested a new experimental approach for the genome-wide identification of insertional retroelement polymorphisms which are characteristic of patients with oncological or autoimmune conditions. This approach was used for the first genome-wide screen and analysis of species-specific and polymorphic integrations of retroelements. This method is also being used for a study of the effect of retroelements on gene expression (Shemyakin and Ovchinnikov Institute of Bioorganic Chemistry, RAS). This line has recently been reorganized and the projects have been included into other lines of research according to their subject.

\section{INNOVATIVE CLINICAL PROJECTS}

A large portion of the projects conducted with support from the program are performed in collaboration with RAS medical facilities. The results of these projects can then be implemented into everyday medical practice. For instance, the following projects were performed in collaboration with the RAS Central Clinical Hospital (Moscow): (a) the study and development of modern methods for the prophylactic and therapeutic treatment of prostate gland cancer using the new drug Mycolicopene; (b) the study of the polysaccharide complexes of mycelial fungi used as dietary fiber for the treatment of diseases of the digestive tract; (c) the development and implementation of the apparatus and software for the Vertikal diagnostic and therapeutic complex used for restoring the ability to walk; (d) the development and implementation of laser-induced hyperthermia of the thyroid gland in the treatment of the nodular goiter; (e) the development and implementation of methods for an individual diagnosis of oxygen side-effects, in which these effects are assessed in combination with the drug therapy; (f) the development and implementation of guidelines for equipping medical diagnostic thermographic facilities, including mobile varieties; ( $g$ ) the implementation of immunochromatographical test-systems based on polymer membranes for the complex express diagnosis of viral hepatitis; and (h) the development of a complex of software and equipment for the $3 \mathrm{D}$ visualization of pathological zone images obtained from endoscopy of the small pelvis organs. One of the most important parts of the program is the creation of an integrated information system for medical facilities. Researchers of the program are the first to use the television method for noncontact endoscopic measurement of the linear parameters of the intracavity objects. The main principles on which integrated medical systems should be based have also been laid down. Specialists have also developed a concept model of a general electronic medical record.

Thus, the "Basic Science for Medicine" Program uses the resources of the eight branches of RAS and its regional branch institutes. It makes an important contribution into the study of the etiology and pathogenesis of socially important diseases, the development and implementation of diagnostic methods, the creation of medical instrumentation and materials, and a search for new medical technologies and drugs. Overall, this program strengthens Russian science and healthcare.

This article is dedicated to the memory of academy member O.G. Gazenko, one of the organizers and ideologists of the program and the constant Chairman of the Scientfic Council.

\section{COMMENTARY FROM THE CURATORS OF INDIVIDUAL LINES OF RESEARCH OF THE BASIC RESEARCH PROGRAM “BASIC SCIENCE FOR MEDICINE" OF THE RAS PRESIDIUM}

RAMS academy member Yurii Vladimirov Andreevich, curator of the Biofotonika line:

The "Biophotonics" line appeared because there is a need for and the possibility of using modern physical and engineering methods involving photonics for the diagnosis and treatment of human diseases. These methods included (1) the creation of various powerful lasers; (2) the creation of sensitive light sensors, both integral and with spatial resolution (matrixes); and (3) the development of computer technology for creating $3 \mathrm{D}$ images of living objects of macroscopic (tomography) and microscopic size (confocal microscopy). 
The aim of this project was to coordinate research in this field and develop new medical technology based on these scientific achievements.

The first step was to inventory all the research on biophotonics by collecting and analyzing bids from laboratories in academic institutes. A large portion of these bids were supported, and initially the decision was almost solely made based on the general scientific quality of the projects. This yielded a very wide variety of projects; however, some projects were good science, but they did not seem to result in the creation of a specific product. After the coordination council was formed, the selection procedure and control of the work procedures became much more stringent. Specifically, a group of qualified independent experts on biophotonics from non-RAS affiliated institutes, including universities and medical institutions, were asked to participate. The overall number of experts was fairly large, so each expert ended up reviewing only $3-4$ projects. The expert assessment was completely anonymous, the experts had no contact with each other, and their names were kept secret. Experience showed that, nevertheless, the opinion of $2-3$ experts that received a project for review was often similar, so the Council did not have much problem making an unambiguous decision on the funding of a project. We also introduced a procedure for reviewing and discussing every project no less than twice a year, one of which took place during a large scientific conference or symposium. In 2008, after numerous discussions, the members decided upon a coordinated plan for further research which would include the following aims: the development of laser technologies for visualizing living structures, the creation of light-consuming nanomaterials for the photothermic destruction of cancer cells in patients and for biochemical analysis using plasmon resonance of nanoparticles, and the development of new sensitizing materials for the photodynamic laser therapy of cancer and for controlling its efficiency.

The "Basic Science for Medicine" program is interesting not only because of its achievements, which are important for both practical and basic science. This program shows the ef- fectiveness of the new principles that underlie the work of the Academy of Sciences. These include democratic, rational, and effective channeling of funds into groups of scientists which have applied for funding, with the independent expert assessment of the projects on one hand and science-based planning of the projects and stringent control over the completion of the projects in all their stages.

\section{RAS member Nikolai Alexandrovich Kuznetsov, curator of the Informatika} line:

The RAS Presidium program "Basic Research for Medicine" coordinates the work of the institutes which belong to eight branches of RAS, as well as many institutes affiliated with the Russian Academy of Medical Sciences and educational institutions which are collaborators in the projects. This means that the importance of the Program is incredibly high. Let us see an example of this in the work of the Biomedical Informatics section. All the work in this line of research can be divided into two subclasses: (1) expert systems made for the diagnosis and prediction of treatment results and (2) computer genomics.

A wide range of expert (partner) systems for a large number of diseases are being developed for computer support of diagnostic and prognostic solutions. The central idea of such systems is the summation, generalizing, and sharing of the experience of highly qualified specialists by using information that has been accumulated by healthcare institutions and medical and biological research centers. This information includes data on patient examination and polling, as well as data on the study of various diseases and therapeutic schemes. The development of software for such expert systems requires the use of a whole range of mathematical methods, such as algorithm theory, recognition theory, mathematical logic, and evaluation and control in indeterminate conditions. The methods, algorithms, and software for these systems are mainly developed in RAS-affiliated institutes, such as the Federal Scientific Center of Russian Federation (RF), the Institute of Medicobiological Problems, RAS; the Computational
Center of the RAS; the Institute of Applied Mathematics, RAS; Harkevich Institute of Information Transmission Problems, RAS; Kotelnikov Institute of Radiotechnics and Electronics, RAS; Institute of Program Systems, RAS; Trapeznikov Institute of Control Sciences, RAS; Institute of Calculation Mathematics, RAS; Institute of Calculation Technology, RAS; Institute of Automation and Electronics, Siberian Branch, RAS; Institue of Image Processing Systems, Siberian Branch, RAS; St. Petersburg Institute of Informatics, RAS; and in the universities and institutes affiliated with the Ministry of Education and Science of the RF:

The Moscow State University; St. Petersburg University; Moscow Institute for Physics and Technology; Bauman State Technical University, Moscow; etc., in conjunction with Russian Academy of Medical Sciences affiliated institutes, institutes of the biological section of RAS, and other medical and healthcare institutions.

In the field of computer genomics, projects include studies of genes and regulatory sites, the prediction of RNA structure, annotations of genes and genomes, an analysis of regulatory signals, an analysis of gene expression, modeling metabolic and regulatory networks, and rational drug development. These projects incorporate a wide spectrum of mathematical methods and various computational equipment, from PCs to supercomputers. These projects are performed in the institutes of the Russian Academy of Sciences, such as the Vavilov Institute of General Genetics, RAS; Engelhardt Institute of Molecular Biology, RAS; Institute of Protein, RAS; Institute of Cytology and Genetics, Siberian Branch, RAS; Harkevich Institute of Information Transmission Problems, RAS; Institute of Mathematical Problems in Biology, RAS; Institute of Cytology of RAS (St. Petersburg), Moscow State University; and St. Petersburg Polytechnical University, as well as universities and institutes affiliated with the Federal Healthcare and Social Development Agency, including the Institute of Physico-Chemical Medicine (RosZdrav) and State Research Institute of Genetics and Selection of Industrial Microorganisms. 
The list of universities and institutes which are involved in biomedical informatics research is very long and is still incomplete. It is presented here to show the importance of coordinating research in this field. Unfortunately, because of the limited funds of the RAS "Fundamental Science for Medicine” Program, only eight projects are currently being supported, so the coordination efforts of the program are limited as well. Real coordination of research in this field is accomplished at the level of seminars, symposiums, and conferences initiated by the Council of the Program. Effective coordination of fundamental research in the medical field requires that the "Basic Science for Medicine" program be awarded interdisciplinary status and receive a considerable increase in funding.
Yurii Vladimirovich Obukhov, doctor of physico-methematical sciences, curator of the Pribory line

Institutes of the Russian Academy of Sciences have performed active research and developed numerous solutions for medical use since the 1970 s. New methods such as laser treatment, radiophysical methods of diagnosis and therapy, magnetic cardiography, encephalography, and other innovative medical technologies have been implemented under the leadership of academy members A.M. Prokhorov, N.D. Devyatkov, Yu.V. Gulyaev, and others. By the mid-1990s, the development of medical instrumentation in the RAS became a very wide field of research and development, which is why the creation of RAS Presidium "Basic Science for Medicine" program was needed, so as to concentrate efforts and funds on the most important lines of basic research and innovation technologies in medical instrumentation.

Currently, the instrumentation section has 14 projects. These are conducted in six RAS organizations in Moscow; two institutes in St. Petersburg; the Pushinskii, Troitskii, and Noginskii Research Centers (four institutes); and in Kazan and Nizhniy Novgorod. This does not include projects conducted in the regional branches of the RAS. The projects are also conducted in conjunction with medical organizations, of which there are more than 30 . The instruments and methods are subjected to trials in the clinics. The results of the projects have been presented at international and Russian conferences and demonstrated at specialized exhibitions. 